\title{
Den Gewissensentscheid des Praktikers akzeptieren
}

Wenn der Tod nicht unerwartet oder gewaltsam eintritt, ist er ein Prozess, der sich über wenige Stunden bis einige Wochen erstrecken kann. Während dieser ganzen, sehr besonderen Phase setzt sich der Sterbende mit seiner Vergangenheit und seinem Werden auseinander, und er sollte dies in einer adäquaten Umgebung und in körperlicher und seelischer Ruhe tun können. Es wäre auch wünschenswert, dass er sich in einem möglichst friedlichen Umfeld von seinen Angehörigen und seinem Leben verabschieden kann.

Leider wird diese so ersehnte Ruhe durch verschiedene Sorgen gestört wie z.B. Schmerzen, Einsamkeit, Abhängigkeit vom Umfeld, den Angehörigen und vom Pflegeteam. Die Begleitung des Sterbenden erfordert eine von Mitgefühl geprägte Unterstützung und eine adäquate palliative Betreuung; die in der heutigen Ausgabe der Schweizerischen Ärztezeitung publizierten medizinisch-ethischen Richtlinien zur «Betreuung von Patientinnen und Patienten am Lebensende» legen denn auch besonderes Gewicht auf diese Punkte.

Die Schweizerische Akademie der Medizinischen Wissenschaften ist so weit gegangen, in diesen besonderen Umständen am Lebensende den Gewissensentscheid eines Arztes, welcher der Bitte eines Patienten nach Suizidbeihilfe nachkommen will, zu respektieren. Dies gilt allerdings nur für den Einzelfall und nur unter Einhaltung gewisser Bedingungen. Dieses Kapitel der Richtlinien wurde sowohl in der Kommission als auch im Rahmen des Vernehmlassungsprozesses ausführlich diskutiert; wie M. Zimmermann und M. Salathé in ihrem Beitrag erwähnen, wurde dieser Abschnitt aus Gründen der Klarheit und Präzision teilweise auch neu formuliert.

Mit der Publikation der definitiven Fassung der Richtlinien «Betreuung von Patientinnen und Patienten am Lebensende» (vom Senat der SAMW verabschiedet an seiner Sitzung vom 25. November 2004) hat die Zentrale Ethikkom- mission ZEK für eine weitere Gruppe von besonders verletzlichen bzw. schutzbedürftigen Menschen den Reflexionsprozess über die adäquate Behandlung und Betreuung abgeschlossen. In ähnlicher Weise veröffentlichte sie - erstmals oder in stark überarbeiteter Form - folgende medizinisch-ethischen Richtlinien:

- Ausübung der ärztlichen Tätigkeit bei Inhaftierten;

- Behandlung und Betreuung von älteren, pflegebedürftigen Menschen;

- Behandlung von zerebral schwerstgeschädigten Langzeitpatienten.

Namentlich in den beiden letztgenannten Richtlinien wurde versucht, durch eine Vereinheitlichung der Struktur die Lektüre zu vereinfachen. Ausserdem ist in mehreren Richtlinien jeweils ein Kapitel der palliativen Betreuung gewidmet. In diesem Zusammenhang haben die Mitglieder der ZEK und der Subkommissionen festgestellt, dass es nötig ist, «palliative Betreuung» besser zu definieren, und zwar in einem konsensuellen Verfahren, bei dem medizinische Fachpersonen aus allen Bereichen beigezogen werden. Die ZEK hat deshalb eine entsprechende Subkommission eingesetzt; diese ist seit September 2003 an der Arbeit und wird voraussichtlich im Laufe dieses Jahres eine erste Fassung der Richtlinien «Palliative Care» vorlegen.

Wir möchten an dieser Stelle allen Mitgliedern der Subkommission und speziell ihrem Präsidenten, Dr. Markus Zimmermann-Acklin, sowie lic. iur. Michelle Salathé, die für das Sekretariat zuständig war, unseren grossen Dank für die geleistete Arbeit aussprechen.

Prof. Michel B. Vallotton, Präsident der Zentralen Ethikkommission

Prof. Peter M. Suter, Präsident der Schweizerischen Akademie der Medizinischen Wissenschaften 


\section{Respecter le choix d'un praticien}

La mort, lorsqu'elle ne survient pas de manière inopinée ou violente, est un processus qui peut s'étendre de quelques heures à quelques semaines jusqu'au décès. Durant toute cette période bien particulière, l'être humain est livré à des pensées et des réflexions sur son passé et son devenir et il devrait bénéficier d'un encadrement adéquat ainsi que d'une quiétude aussi bien physique que psychique. Dans ce même ordre d'idées, il doit aussi pouvoir prendre congé de ses proches et de la vie, dans une ambiance aussi sereine que possible.

Malheureusement, cette sérénité tant désirée est souvent gâchée par des préoccupations telles que souffrances, isolement, dépendance physique de l'entourage, des proches et de l'équipe de soins médico-infirmiers. L'accompagnement du mourant requiert une sollicitude empreinte de compassion et un soutien palliatif approprié, d'où l'accent mis sur ces points dans les présentes directives.

Dans ces conditions spécifiques de fin de vie, l'Académie Suisse des Sciences Médicales est allée jusqu'à admettre respecter le choix d'un praticien à accéder, en toute conscience personnelle, à la demande d'aide au suicide d'un patient dans des situations bien particulières, soumises à des conditions formelles. Tant en commission qu'au cours du processus de consultation, ce chapitre des directives a été longtemps débattu et il a été reformulé pour gagner encore en clarté et en précision, comme en témoignent Mme Salathé et M. Zimmermann-Acklin dans leur article.

Les directives sur la Prise en charge des patientes et patients en fin de vie, ont été approuvées dans leur version finale par le Sénat de l'Académie lors de sa séance du 25 novembre 2004. La Commission centrale d'éthique a ainsi parachevé une réflexion en profondeur portant sur l'exercice de la médecine et des soins infirmiers auprès de cette catégorie de patients représentant une population vulnérable et fragilisée.
Les directives précédemment citées s'ajoutent à trois autres déjà existantes sur des thèmes assez proches, soit:

- l'Exercice de la médecine auprès de personnes détenues;

- le Traitement et la prise en charge des personnes âgées en situation de dépendance;

- mais encore sur le Traitement et la prise en charge des patient(e)s souffrant d'atteintes cérébrales extrêmes de longue durée.

Le lecteur remarquera notamment qu'une harmonisation de la structure de ces textes a été réalisée afin d'en faciliter la consultation. Mais encore, dans plusieurs directives, un chapitre est consacré aux soins palliatifs qui devraient être envisagés, proposés et appliqués suffisamment précocement en sus et en parallèle des soins curatifs. Les membres de la Commission centrale d'éthique et de ses sous-commissions ont ainsi pu constater la nécessité de mieux définir, et de manière consensuelle, ce que les soins palliatifs représentaient, en prenant en considération tous les acteurs des équipes de soins médicaux.

Raison pour laquelle la Commission centrale d'éthique a créé en 2003 une nouvelle sous-commission qui s'est rapidement mise au travail et qui présentera courant 2005 un texte mis à jour des directives sur les soins palliatifs.

Que tous les membres de la sous-commission et tout particulièrement son président, le Docteur Markus Zimmermann-Acklin, ainsi que Madame Michelle Salathé qui en a assuré le secrétariat, trouvent ici l'expression de notre profonde reconnaissance pour le travail accompli.

Prof. Michel B. Vallotton, Président de la Commission centrale d'éthique

Prof. Peter M. Suter, Président de l'Académie Suisse des Sciences Médicales 